\title{
Rhabdomyolysis and Acute Pancreatitis in Diabetic Ketoacidosis: A Case Report and Review of Literature
}

\author{
Muhammad Umair ${ }^{1}$, Sanna Salam ${ }^{*}\left(\mathbb{D}\right.$, Anjum Kazi², Almeet Kaur ${ }^{2}$, \\ Ardriana Abrudescu' ${ }^{1}$, Theo Trandafirescu ${ }^{1}$ \\ ${ }^{1}$ Internal Medicine Department, Icahn School of Medicine of Mount Sinai Queens Hospital Centre, New York, NY, USA \\ ${ }^{2}$ NYIT Collage of Osteopathic Medicine, New York, NY, USA \\ Email: *drsanama_leo@yahoo.com
}

How to cite this paper: Umair, M., Salam, S., Kazi, A., Kaur, A., Abrudescu, A. and Trandafirescu, T. (2021) Rhabdomyolysis and Acute Pancreatitis in Diabetic Ketoacidosis, A Case Report and Review of Literature. Open Journal of Endocrine and Metabolic Diseases, 11, 161-164.

https://doi.org/10.4236/ojemd.2021.119014

Received: August 17, 2021

Accepted: September 20, 2021

Published: September 23, 2021

Copyright $\odot 2021$ by author(s) and Scientific Research Publishing Inc. This work is licensed under the Creative Commons Attribution International License (CC BY 4.0).

http://creativecommons.org/licenses/by/4.0/

\begin{abstract}
Diabetic ketoacidosis (DKA) is a life-threatening complication in patients with Type 1 or Type II. Diabetes Mellitus resulting in fluid shifts, electrolytes imbalance and acid-base disorders, can lead to multi-organ Failure. The Pancreas and skeletal muscles are not commonly affected in DKA. We present a case of 41-year-old female who was initially admitted in intensive care unit for treatment of Diabetic ketoacidosis, and was treated appropriately later she complained of abdominal pain and generalized myalgia. Hospital course was complicated with Acute pancreatitis and Rhabdomyolysis. Serum electrolytes were significant for hypo-phosphatemia. Workup for myositis and acute pancreatitis were negative. The exact etiology is unknown, but it is proposed in many literature articles that serum hyper-osmolarity and/or electrolyte abnormalities can be the culprits in triggering the complications.
\end{abstract}

\section{Keywords}

Insulin Dependent Diabetes Mellitus (IDDM), Acute Pancreatitis, Rhabdomyolysis, Acute Kidney Injury

\section{Introduction}

Diabetic ketoacidosis (DKA) is a life-threatening complication in patients with Type 1 or Type II Diabetes Mellitus. The Pancreas and skeletal muscles are not commonly affected in DKA but fluid shifts along with changes in the serum osmolarities, electrolyte imbalance, and acid-base disorders can lead to multi-organ dysfunction such as the one presented here. The exact etiology is unknown, but it 
is proposed in many literature articles that serum hyper-osmolarity and/or electrolyte abnormalities can be the common culprits in triggering the complications.

\section{Case Presentation}

We present a case of a 41-year-old female with a past medical history of Ulcerative Colitis, and DKAs along with uncontrolled Diabetes Mellitus presented to the emergency room with complaints of generalized weakness, abdominal pain, and drowsiness for 2 days. At presentation, she was afebrile, with BP of 133/76 $\mathrm{mmHg}$, HR of $80 \mathrm{bpm}$, and oxygen saturation of $98 \%$. The physical exam was unremarkable other than mild confusion and lethargy. Respiratory, cardiac, and abdominal exams were unremarkable. Basic blood workup showed Hemoglobin of $18 \mathrm{gm} / \mathrm{dL}$, White Blood Cells: $20.4 \times(10)^{3} / \mathrm{mcL}$, Platelets count of 188,000 . Basic metabolic panel showed serum Sodium of $157 \mathrm{mmol} / \mathrm{L}$, Potassium 4.2 $\mathrm{mmol} / \mathrm{L}$, bicarbonate $17.2 \mathrm{mmol} / \mathrm{L}$, and Lactate of $5 \mathrm{mmol} / \mathrm{L}$. The blood glucose level was $1400 \mathrm{mg} / \mathrm{dL}$ with a serum osmolarity of $413 \mathrm{mOsm} / \mathrm{kg}$. Venous blood gas showed a pH of 7.225 with an Anion gap of $36 \mathrm{mEq} / \mathrm{L}$ leading to the diagnosis of anion gap metabolic acidosis suggestive of DKA. Pt was admitted to the Intensive Care Unit for closer monitoring and management. Intravenous fluids and IV insulin were started. Serum electrolytes were closely monitored. During hospitalization, the patient started complaining of generalized body aches and myalgias. Creatinine phosphokinase (CPK) was elevated with the lab value of 20,000 U/L along with elevated CK-MB. Serum phosphorus dropped from the initial normal to 1.5 , which improved after replacement. Extensive workup to rule out infectious and inflammatory causes of myositis along with muscle biopsy was done but did not show any evidence of inflammatory or infectious myositis. Serum Amylase and Lipase were checked in the setting of persistent abdominal pain and resulted in $145 \mathrm{U} / \mathrm{L}$ and $370 \mathrm{U} / \mathrm{L}$ respectively. Abdominal Ultrasound, CT \& MRI abdomen \& pelvis, and Magnetic Resonance Cholangiopancreatography (MRCP) were performed and indicated acute pancreatitis (AP) with fluid collection without evidence of gallstones. Meanwhile, DKA was resolved and the patient's condition improved with conservative management, then transferred to the medicine floors and was subsequently discharged with the followup as an outpatient in the clinic.

\section{Discussion}

Diabetic ketoacidosis (DKA) can be life-threatening in patients with uncontrolled Diabetes Mellitus (DM) especially in patients with type II Diabetes Mellitus. Acute pancreatitis (AP) and non-traumatic rhabdomyolysis can occur in severe cases of DKA which can increase the mortality if left undetected [1]. Rhabdomyolysis is a syndrome characterized by myonecrosis and the release of intracellular muscle constituents i.e. Creatinine Kinase (CK) enzymes and myoglobin into the circulation [1]. The severity ranges from asymptomatic cases to life- 
threatening manifestations associated with extreme enzyme elevations, electrolyte imbalances, and acute kidney injury [1]. Common clinical symptoms are muscle pain, dark urine, and generalized weakness. Rhabdomyolysis is classified into three groups: 1) traumatic; 2) non-traumatic exertional; and 3) non-traumatic non-exertional [1]. Although the exact mechanism of action of a hyperosmolar state causing rhabdomyolysis remains unclear, there have been theories postulating the etiology. One possibility is that hyperosmolarity can damage the myocytes, also serum electrolyte abnormalities such as Hypernatremia, Hypokalemia, and Hypophosphatemia seen in hyperosmolar hyperglycemic states can also contribute to rhabdomyolysis. There is a rapid shift of electrolytes especially Potassium and Phosphate from extracellular to intracellular with intravenous insulin infusions in diabetic emergencies. Rhabdomyolysis itself is known to cause hypokalemia in isolated cases as well.

In the review of the literature, Amin et al. [1] presented a case of Rhabdomyolysis-Induced acute kidney injury in DKA and emphasized the necessity to rule out other causes of renal failure in patients admitted for Diabetic emergencies to decrease morbidity and mortality. Al-Azzawi et al. [2], stated Rhabdomyolysis as an underdiagnosed complication of DKA and emphasized that the serum electrolyte abnormalities have significant implications on the recovery of patients. A cross-sectional study was carried out in the emergency department of Baghdad, where 43 patients with Type 1 Diabetes Mellitus presenting with diabetic ketoacidosis were enrolled. The incidence of rhabdomyolysis was $6.98 \%$ as was seen in 3 cases.

Shah SK et al. [3], presented an interesting case of rhabdomyolysis secondary to hypophosphatemia in the setting of DKA. The possible cause of rhabdomyolysis was attributed to severe hypophosphatemia (minimum serum phosphate, as low as $0.8 \mathrm{mg} / \mathrm{dL}$ ). Wang LM et al. [4], found 44 cases (16.60\%) of Rhabdomyolysis in 265 diabetic emergencies (including DKA or hyperosmolar, or both). The diagnosis was based on 1) serum creatinine kinase (CK) > $1000 \mathrm{IU} / \mathrm{l}$ and 2) the absence of acute myocardial infarction, stroke, and end-stage renal disease. They found the mortality within 1 week of diabetic emergencies (38.5\% for DKA, $35.5 \%$ for HHNS (Hyperosmolar, hyperglycemic nonketoic syndrome) was higher in patients with Rhabdomyolysis than those without (9.7\% for DKA, $26.7 \%$ for HHNS).

In our case, the most common causes of AP were ruled out, including alcoholism, gallstones, hypertriglyceridemia, and autoimmune causes. For the rhabdomyolysis, inflammatory myositis and infectious myositis were ruled out with the help of muscle biopsy leaving hypophosphatemia resulted in the management of DKA as the possible culprit of rhabdomyolysis in DKA

\section{Conclusion}

Due to the high mortality rates associated with rhabdomyolysis and hyperosmolar hyperglycemic syndrome (HHS) and DKA, it is important to establish an early 
diagnosis. Routine screening by ordering CPK levels should be considered in highly suspected patients admitted with diabetic emergencies. Acute kidney injury associated with rhabdomyolysis plays a vital role in the recovery and duration of the hospital stay. Rhabdomyolysis should be suspected in patients presenting with diabetic emergencies and acute kidney injury when common causes have been excluded and treated accordingly without improvement of kidney functions.

\section{Conflicts of Interest}

The authors declare no conflicts of interest regarding the publication of this paper.

\section{References}

[1] Amin, A., Gandhi, B., Torre, S., et al. (2018) Rhabdomyolysis-Induced Acute Kidney Injury in Diabetic Emergency: Underdiagnosed and an Important Association to Be Aware of. Case Reports in Medicine, 2018, Article ID: 4132738. https://doi.org/10.1155/2018/4132738

[2] Al-Azzawi, O.F.N., Razak, M.K.A. and Al Hammady, S.J. (2019) Rhabdomyolysis; Is It an Overlooked DKA Complication. Diabetes \& Metabolic Syndrome: Clinical Research \& Reviews, 13, 3047-3052. https://doi.org/10.1016/j.dsx.2018.07.005

[3] Shah, S.K., Shah, L., Bhattarai, S. and Giri, M. (2015) Rhabdomyolysis Due to Severe Hypophosphatemia in Diabetic Ketoacidosis. JNMA Journal of Nepal Medical As sociation, 53, 137-140 https://doi.org/10.31729/jnma.2777

[4] Wang, L.M., Tsai, S.T., Ho, L.T., Hu, S.C. and Lee, C.H. (1994) Rhabdomyolysis in Diabetic Emergencies. Diabetes Research Clinical Practice, 26, 209-214. https://doi.org/10.1016/0168-8227(94)90062-0 